\title{
Acute Kidney Injury Following Surgical Aortic Valve Replacement
}

Marc Najjar, M.D., Halit Yerebakan, M.D., Robert A. Sorabella, M.D., Denis J. Donovan, B.S., Alexander P. Kossar, B.S., Sowmyashree Sreekanth, M.D., Paul Kurlansky, M.D., Michael A. Borger, M.D., Michael Argenziano, M.D., Craig R. Smith, M.D., and Isaac George, M.D. New York Presbyterian Hospital - College of Physicians and Surgeons of Columbia University, New York, New York

\section{Abstract}

Background-Acute kidney injury (AKI) is a significant complication of surgical aortic valve replacement (SAVR). This study sought to describe AKI following SAVR, its risk factors, predictors and effect on long-term survival.

Methods-We retrospectively reviewed 2169 patients who underwent isolated SAVR between 2000 and 2012. The main end-points were occurrence of AKI, postoperative complications, and short-and long-term survival rates following SAVR. Patients were divided into two groups: AKIR $(\mathrm{n}=181)$ and AKIS $(\mathrm{n}=1945)$.

Results-AKI occurred in $8.5 \%$ of patients, of which $3.9 \%(\mathrm{n}=7)$ needed dialysis. Predictors of AKI after SAVR were body mass index (BMI) and intraoperative packed red blood cells (PRBC) transfusion. AKIR patients had a more complicated postoperative course and higher cumulative mortality $(25 \%$ vs. $17 \%, \mathrm{p}=0.012)$ with a median follow-up of 4.1 years. AKI was not found to be an independent predictor of mortality.

Conclusions-Predictors of AKI after SAVR are increased BMI and intraoperative PRBC transfusion. AKI conferred an increase in hospital length of stay and cumulative mortality while the need for postoperative dialysis was associated with the most complicated hospital stays and the highest in-hospital and cumulative mortalities; therefore careful recognition of patients at risk of AKI is warranted for a better preoperative renal optimization. However, incidence of AKI was lower than what is reported after both on-CPB cardiac surgeries and transcatheter aortic valve replacement, moreover AKI was not found to be an independent predictor of mortality.

Surgical aortic valve replacement (SAVR) is the most common cardiac valve operation in the United States and still the standard treatment for most patients with severe aortic valve

\footnotetext{
Address for correspondence: Isaac George, M.D., Assistant Professor of Surgery, Division of Cardiothoracic Surgery, Columbia University College of Physicians and Surgeons, New York-Presbyterian Hospital, 177 Fort Washington Ave, MHB 7GN-435, New York, NY 10032. Fax: +1-212-305-2439; ig2006@cumc.columbia.edu.

Conflict of interest: None

The manuscript was presented in part as an abstract during the 63rd American College of Cardiology Annual Meeting (Washington DC, March 29, 2014).

SUPPORTING INFORMATION

Additional supporting information may be found in the online version of this article at the publisher's web-site.
} 
disease. ${ }^{1,2}$ Acute kidney injury (AKI) is a well-known and clinically significant complication after cardiac surgery. Over the last decade, the reported incidence of AKI following cardiac surgery has been inconsistent, ranging from $9 \%$ to $43 \%{ }^{3,4}$ depending on the patient population and definition/classification of AKI. Efforts have been made to address this issue as several definitions of AKI have been adopted over the years, namely the RIFLE, the modified RIFLE and, most recently, the acute kidney injury network (AKIN) criteria. ${ }^{5,6}$

The occurrence of AKI exponentially raises postoperative mortality, which, in some series, exceeds $40 \%$ and even reaches $50 \%$ in those patients requiring dialysis compared to mortality rates of $0.6-2 \%$ in patients with no AKI. ${ }^{7-9}$ In fact, changes in postoperative serum creatinine have consistently been associated with a higher risk of infection, a more complicated hospital course ${ }^{10-14}$ as well as a higher mortality even when renal function recovered. ${ }^{15,16}$

We sought to describe the occurrence of AKI following SAVR and the risk factors for its development. We also analyze the impact of AKI on the postoperative course as well as on short- and long-term survival.

\section{MATERIALS AND METHODS}

\section{Patient population}

A total of 2169 consecutive patients undergoing SAVR at New York Presbyterian HospitalColumbia University Medical Center between 2000 and 2012 were studied and clinical outcomes data were collected from chart review and analyzed retrospectively. All patients met standard indications for surgery for aortic stenosis (AS) or aortic insufficiency (AI). The Institutional Review Board of Columbia University approved all study protocols and waived informed consent.

\section{Demographics and outcome measures}

Baseline characteristics (demographic data, comorbidities, hemodynamic variables, and serum laboratory values), operative details as well as postoperative outcomes were collected prospectively for all patients as part of regular patient care.

The main end-points were occurrence of AKI and long-term renal function following SAVR. AKI was defined by AKIN as follows: Stage 1: increase in SCr of 1.5 but $<2$ times or an increase of $0.3 \mathrm{mg} / \mathrm{dL}$ ( $26.4 \mathrm{mmol} / \mathrm{L}$ ) or a urine output $<0.5 \mathrm{~mL} / \mathrm{kg} / \mathrm{h}$ for $>6$ but $<12$ hours; Stage 2: increase in SCr of 2 but $<3$ times or a urine output $<0.5 \mathrm{~mL} / \mathrm{kg} / \mathrm{h}$ for 12 but $<24$ hours; and Stage 3: increase in SCr of 3 times or a SCr of $4 \mathrm{mg} / \mathrm{dL}$ ( $354 \mathrm{mmol} / \mathrm{L}$ ) or a urine output $<0.3 \mathrm{~mL} / \mathrm{kg} / \mathrm{h}$ for $>24$ hours or anuria for 12 hours (Table 1 ). Patients were therefore divided into two groups (AKI+ and AKI) based on the occurrence of AKI in the first seven days postoperatively using the highest postoperative $\mathrm{SCr}$ value within this time period. Estimated glomerular filtration rate (eGFR) was used to assess renal function. eGFR was calculated using the Chronic Kidney Disease Epidemiology Collaboration (CKD-EPI) formula. ${ }^{17}$ Base-line eGFR was determined using the last preoperative $\mathrm{SCr}$ value prior to surgery. Patients who were preoperatively on dialysis $(n=43)$ were excluded from the study 
as their creatinine levels were clinically insignificant and could not be used to assess renal function.

Other outcomes of interest included postoperative complications, intensive care unit length of stay, total hospital length of stay, in-hospital mortality and survival rates at one, three, and five years. Complication and death data were obtained from clinical chart review and was verified by the national social security as well as the institutional death indexes.

\section{Statistical analysis}

Continuous data are expressed as mean standard deviation (95\% confidence interval, [CI]).

Categorical data are presented as percentage and frequency. Baseline, operative, and postoperative characteristics and outcomes were compared between AKI+ and AKI patients using independent-sample Student's t-tests for normally distributed continuous variables and Mann-Whitney U test for non-normally distributed data, while chi-square test with post hoc Fisher's exact test was used for categorical variables. Paired t-test was used to compare eGFR levels over time. Preoperative characteristics within the AKI+ and AKI groups at different follow-up times were compared using independent samples t-testing in order to account for competing risk between the primary outcome and death, as well as ensure homogeneity of patients in the follow-up groups. Mortality analysis and survival over time were depicted using Kaplan-Meier estimate. Survival difference was evaluated using Mantel- Haenszel log-rank test.

To determine independent predictors of AKI, a multivariable logistic regression was used to include variables with $\mathrm{p}$ value $<0.2$ in the univariate analysis of five variables. Predictors of long-term mortality were determined using an adjusted multivariate Cox proportional hazards regression analysis. Adjusted hazard ratios were reported for each variable with $95 \%$ CI. Odds ratios and $95 \%$ CI were reported. p-values of 0.05 were considered statistically significant for both regression and nonregression analyses. All statistical computations were performed using SPSS version 22 (IBM Corp., Armonk, NY, USA).

\section{RESULTS}

\section{Preoperative and operative characteristics}

The baseline characteristics of 2126 consecutive patients undergoing isolated SAVR are summarized in Table 2. Mean age of patients was 70.614 .4 years and $42.9 \%$ were females. Mean follow-up duration was 4.9 3.5 years. Seventy-two percent of patients were operated for AS and $28 \%$ for AI. AKI+ patients had a higher prevalence of chronic obstructive pulmonary disease (COPD) and diabetes (respectively $15 \%$ vs. $9.6 \%, \mathrm{p}=0.022$ and $26 \%$ vs. $20 \%, \mathrm{p}=0.048)$ and had a higher body mass index (BMI) (29 6.4 vs. 28 5.7, p = 0.008). Although not statistically significant, AKI+ patients also tended to be slightly older as compared to AKI patients. Preoperative cardiac catheterization interval was not different between AKI+ and AKI patients with a similar proportion in both groups having had a short interval ( 5 days) (respectively 33 and 30\%, $\mathrm{p}=0.555$ ).

Surgical priority as well as $\mathrm{CPB}$ and cross-clamp (XC) times were similar between $\mathrm{AKI}+$ and AKI patients. The main operative differences between the two groups were increased 
frequency of and need for intraoperative PRBC transfusion in the AKI+ group (40\% vs. $31 \%$ of cases, $p=0.007$ and 1.11 .4 vs. 0.81 .5 units, $p=0.026$ ). Tissue valves were most commonly used in both groups. The operative variables are summarized in Table 3.

\section{Acute kidney injury and kidney function}

Acute kidney injury occurred in 8.5\% of patients (181/2126), 3.9\% (7/181) of which needed acute renal replacement therapy (Table 4). Two-thirds (67\%) of AKI+ patients had stage 1 AKI, $23 \%$ had stage 2 AKI, and only $10 \%$ suffered from stage 3 . AKI+ patients showed an initial acute decline in eGFR postoperatively, reaching its nadir at two days post-SAVR with a value of 43.322 .4 ( $p<0.001$ vs. baseline) (Fig. 1). Thereafter, eGFR showed an improvement over time, which was maintained up to 12 months postoperatively with an eGFR of 59.723 .6 , a value still significantly higher than the postop period ( $p<0.05$ vs. 2 days). However, despite this gradual and steady improvement, kidney function did not, over the follow-up period, go back to the baseline levels. At 12 months postoperatively, eGFR was still significantly lower than at baseline (59.7 23.6 vs. $70.723 .0, p=0.017)$. On the other hand, AKI patients showed an initial improvement in eGFR post SAVR, which reached a peak of 75.023 .0 at one week postop ( $p<0.001$ vs. baseline). Thereafter eGFR decreased and stabilized from one month to 12 months at 65.523 .0 , a level close to and not statistically different from baseline (65.5 23.0 vs. $69.221 .2, \mathrm{p}=0.401$ ). AKI patients had consistently higher eGFR levels at all time points following SAVR compared to AKI+ patients (67.4 24.3 vs. 56.025 .9 at one month; 66.923 .7 vs. 58.719 .3 at six months and 65.423 .0 vs. 59.723 .6 at 12 months, $\mathrm{p}<0.001)$.

In order to ascertain that improvement in kidney function is not caused by a selection bias related to the death of the sickest patients with AKI, we analyzed competing risk by comparing representative risk in patients at follow-up versus the baseline cohort. Independent samples t-test was used to compare patients within each AKI group (AKI+ and AKI ) at different follow-up times with the baseline cohort in terms of preoperative characteristics, namely age, gender, BMI, preoperative LVEF, preoperative eGFR, reoperative cardiac surgery, and preoperative comorbidities such as CVD, PVD, COPD, and diabetes (Tables S1 and S2). We found that the follow-up patients at all time points in both the AKI+ and AKI groups displayed equivalent comorbidities and were not younger and healthier than the baseline population, suggesting that these patients at follow-up are representative of the general cohort and that death did not confound the survival analysis interpretation.

Looking at the pre- and intraoperative factors that were different between $\mathrm{AKI}+$ and $\mathrm{AKI}$ patients (diabetes, BMI, and intraoperative PRBC transfusion), we found that AKI occurred more often in obese versus nonobese patients ( $11 \%$ vs. $8 \%, p=0.029)$, in diabetic versus nondiabetic patients $(11 \%$ vs. $8 \%, \mathrm{p}=0.048)$, and in patients who received intraoperative PRBC transfusion versus those who were not transfused ( $11 \%$ vs. $7 \%, \mathrm{p}=0.007)$ (Table S3). Predictors of AKI were determined using a logistic multivariable regression, and included variables with a $\mathrm{p}<0.02$ on univariate analysis. BMI (OR 1.032, CI 1.007-1.057, $\mathrm{p}=0.013$ ) and intraoperative PRBC transfusion (OR 1.094, CI 1.006-1.191, $\mathrm{p}=0.037$ ) were significant predictors of AKI after SAVR (Table 5). 


\section{Postoperative complications}

In general, patients suffering postoperatively from AKI had more complicated postoperative hospital courses. Intensive care unit length of stay (ICULOS) and total hospital lengths of stay (THLOS) were significantly longer for the AKI+ patients (respectively, 4.25 .3 vs. 2.6 3.7 days, $\mathrm{p}<0.001$ and 1513 vs. 109.3 days, $\mathrm{p}<0.001$ ) (Table 4). In addition, $11 \%$ of AKI + patient were readmitted to the ICU during their hospital stay compared to only $3 \%$ of AKI patients ( $p<0.001)$. Finally, $2.2 \%$ of AKI+ patients suffered from sepsis versus only $0.5 \%$ of AKI ( $\mathrm{p}=0.026)$. There was no difference among the groups in terms of other complication or in-hospital mortality. Postoperative complications are summarized in Table 4.

Among AKI+patients, more severe stages of AKI were associated with longer and more complicated hospital stays. Average ICULOS and THLOS among stages 1, 2, and 3 AKI patients were, respectively, $4.05 .1,3.73 .5$, and 7.48 .8 days, $\mathrm{p}=0.046$ and $14.513 .7,12.9$ 9.5 and 20.717 .2 days, $\mathrm{p}=0.151$ (Table S4).

Seven patients (3.9\%) in the AKI+ group required acute RRT postoperatively (RRT+). Compared with AKI+ patients who did not require acute RRT (RRT ), RRT+ patients had a more complicated postoperative course with a higher rate of complications and longer ICULOS and THLOS (respectively 7.7 4.1 vs. 4.15 .3 days, $\mathrm{p}=0.012$ and 36.933 .5 vs. 13.8 11.2 days, $\mathrm{p}=0.005)($ Table $\mathrm{S} 5)$.

\section{Survival outcomes}

Thirty-days mortality was not significantly different between AKI+ and AKI-patients (respectively $2.8 \%$ vs. $1.5 \%, \mathrm{p}=0.215$ ); however AKI + patients had a higher cumulative mortality $(25 \%$ vs. $17 \%, \mathrm{p}=0.012)$ with a median follow-up of 4.1 years. As seen in Figure 2 , there were slight differences in one, three, and five-year survivals between the two groups $(\log$-rank $\mathrm{p}=0.002$,$) . Among patients who died, there was a trend towards a higher rate of$ diabetes among AKI+ patients ([29\% $\{13 / 45\}$ vs. $17 \%\{58 / 338\}$ p $=0.067])$.

In order to identify independent predictors of all-cause mortality, a multivariable Cox regression was performed using pre-, intra-, and postoperative risk data, and with adjustment for age and gender (Table 6). Age (HR = 1.050, CI 1.037-1.062, $\mathrm{p}<0.001), \mathrm{CVD}(\mathrm{HR}=$ 1.465, CI 1.079-1.988, $\mathrm{p}=0.014)$, COPD (HR = 1.801, CI 1.349-2.406, p 0.001), preoperative LVEF $(\mathrm{HR}=0.985$, CI 0.978-0.993, $\mathrm{p}<0.001)$, intraoperative RBC transfusion $(\mathrm{HR}=1.319, \mathrm{CI} 1.030-1.689, \mathrm{p}=0.028)$, postoperative need for dialysis $(\mathrm{HR}=$ 5.091, CI 1.554-16.673, $\mathrm{p}=0.007)$, ICULOS (HR = 1.024, CI 1.006-1.042, $\mathrm{p}=0.007$ ), and postoperative readmission to ICU $(\mathrm{HR}=2.715$, CI $1.852-3.979, \mathrm{p}<0.001)$ were found to be independent predictive risk factors for survival. The occurrence of AKI postoperatively was not found to be an independent predictor of mortality on multivariable Cox regression (HR = 1.127, CI 0.805-1.580, $\mathrm{p}=0.486$ ).

More severe AKI stages tended to be associated with higher mortality rates although the results lack statistical significance. Stages 1, 2, and 3 AKI patients had in-hospital and cumulative mortality rates of, respectively, 2, 5, and 6\%, p $=0.188$ and 23, 24, and 39\%, p = 0.125 (Table S4). 
Finally, the need for postoperative acute RRT conferred a clear mortality risk in the AKI+ patients. In fact, both in-hospital and cumulative mortalities were significantly higher in the RRT+ group compared to the RRT group (respectively, $2 \%$ vs. $29 \%, p=0.012$ and $23 \%$ vs. $71 \%, \mathrm{p}=0.011)($ Table S5).

\section{CONCLUSIONS}

Acute kidney injury is a well-recognized complication of cardiac surgery and is known to contribute to several complications such as longer hospital stay, increased risk of infection, sepsis, and postoperative mortality. Although valve surgery has been shown to be an independent risk factor for AKI with a 2.7 increased risk compared to coronary artery bypass grafting (CABG), ${ }^{9} \mathrm{AKI}$ in the setting of isolated SAVR has not been described. This relationship is even of greater importance with the advent of TAVR, its increased availability and expanding potential patient population. In fact, TAVR itself has been strongly linked with the development of AKI, especially contrast-induced nephropathy (CIN), a complication that could contraindicate TAVR in patients with borderline renal function. Therefore, adding the risk of AKI as a variable in the decision-making between SAVR and TAVR deserves close attention.

We sought, in the present study, to report the incidence, determine the predictors, and examine the outcomes of AKI following isolated SAVR in a large single center. The primary findings were as follows: 1) AKI occurred in $8.5 \%$ of patients following SAVR, 2) AKI+ patients had diabetes and COPD more often at baseline and received more blood transfusions in the perioperative period, 3) AKI+ patients had a more complicated postoperative course with longer THLOS and ICULOS, higher rates of sepsis and more frequent readmissions to the ICU, 4) independent predictors of AKI included BMI and higher intraoperative blood transfusion rate, 5) AKI+ patients did not have a shorter preoperative cardiac catheterization interval, 6) AKI+ and AKI patients had a similar inhospital mortality but AKI+ patients had a higher cumulative mortality, 7) AKI was not found to be an independent predictor of mortality, and 8) RRT+ patients had longer ICULOS and THLOS and higher in-hospital and cumulative mortalities compared to RRT patients.

AKI occurred in $8.5 \%$ of patients after SAVR, of which 3.9\% required dialysis. These numbers are lower than what have been previously reported in the cardiac surgery literature. In fact, recent series looking at AKI following CPB cardiac surgery using the AKIN criteria have reported AKI incidence rates ranging from $8.9 \%$ to $39 \%$.,7,10,18 The largest of these series came from Robert et al. ${ }^{18}$ who reported 30\% incidence of AKI in 25,086 patients undergoing cardiac surgery. Again, the main difference with our study is the type of surgery, as the latter series included $68.9 \% \mathrm{CABG}$ and only $15.6 \%$ of isolated valve surgeries. These findings highlight a possible difference in AKI incidence among different cardiac surgeries. ${ }^{9}$

We report similar results to previous studies in various cardiac surgeries regarding predictors of AKI and postoperative course after AKI. Karkouti et al. ${ }^{19}$ found that both intraoperative blood transfusion and BSA are predictors of AKI, whereas Bove et al. ${ }^{20}$ also reported blood transfusion as being a risk factor for AKI. The relationship between PRBC transfusion and the development of AKI has long been reported in the surgical literature. The need for blood 
transfusion could have a multidimensional relation with kidney function; on one hand, it might signify increased blood loss and thus a higher probability for a hypotensive state, which is a main pathogenic mechanism of AKI. On the other hand new evidence suggests that storage causes structural changes to PRBCs leading to their release of proinflammatory molecules and procoagulant lipids, all of which can contribute to a direct injury to the kidneys. ${ }^{4,19,21}$ Moreover, we report a more complicated postoperative course for AKI+ patients with higher infection rates and significantly longer ICULOS and THLOS. Similar findings were reported by several studies, especially the increased lengths of stays. ${ }^{4,10,22}$ Postoperative AKI has been also linked with worsened survival rates in most studies; in some series AKI+ patients have 30-day mortalities up to 10 times higher than AKI patients. ${ }^{7,23}$ However, our data did not show any statistically different in-hospital mortality between the two groups, and only the cumulative mortality was higher in the AKI+ patients. In our study, one, three, and five-year survivals were lower in AKI+ compared to AKI patients, but this difference, although statistically significant, was not large, as seen in Figure 2. Importantly, using a logistic regression model, AKI was not found to be an independent predictor of mortality, which casts doubt on the strength of association between AKI and mortality after SAVR. This finding could be attributed in part to the presence of more comorbidities in AKI+ patients as compared to AKI but possibly also to the relatively low incidence of AKI in our cohort. The low incidence of AKI might be explained by the complex relationship of SAVR and kidney function: the risk of AKI may be offset by the beneficial effect of SAVR improving forward flow and organ perfusion. It should be noted that the incidence of AKI after AVR is lower than other cardiac surgeries and may have led to a weaker association between AKI and mortality.

We believe that a careful screening of patients should be done preoperatively in order to identify those patients most at risk of developing AKI. Once identified, several preventive measures can be taken to reduce the incidence of AKI, including: 1) optimization of renal perfusion through perioperative hydration, careful hemodynamic monitoring, and the use of inotropic agents, 2) discontinuation of nephrotoxic medications such as NSAIDs, and although still a source of debate, the discontinuation of ACE inhibitors and ARBs is also advisable, ${ }^{24-26}$ and 3) early pharmacological interventions using Fenoldopam (selective DA-1 agonist, increase in renal blood flow) or atrial natriuretic peptide (natriuresis) have been shown to reduce the incidence of AKI. ${ }^{27,28}$ Furthermore, intraoperative blood transfusion, one of the few modifiable risk factors, was found in the current study to be a significant predictor of both AKI as well as mortality—we strongly recommend careful surgical hemostasis as well as judicious blood transfusion only when necessary to potentially reduce the risk of AKI. We did not find that a shorter preoperative cardiac catheterization interval ( 5 days) is associated with a higher AKI incidence in isolated SAVR. These results reiterate the conclusions of other research groups who found that delaying isolated valve surgeries after coronary angiography is not justified in most patients. ${ }^{30-32}$ Whenever AKI occurs, efforts should focus on providing an early diagnosis and treatment plan, especially that duration of AKI has been shown to impact long-term survival following cardiac surgery. ${ }^{10}$ Some major therapeutic principles are: 1) maintenance of systemic hemodynamics through the detection and treatment of volume depletion, improvement of cardiac contractility using inotropes, and reduction of afterload with 
vasodilators, 2) avoidance of nephrotoxic drugs and agents, 3) use of diuretics such as furosemide ${ }^{29,30}$ to increase urine output, 4) mesenchymal stem cells (MSC), a novel and promising therapeutic option whose benefits are related to the release by MSCs of factors promoting tubular cell proliferation, ${ }^{33-35}$ and if all fails to improve renal function, 5) early renal replacement therapy can be attempted, as it has shown promising results in some series. $^{36-39}$

Although it does not involve CPB with its hemodynamic instability and systemic inflammatory reactions, TAVR poses a significant risk to the kidneys as well. Some of the major pathogenic factors contributing to AKI after TAVR are the use of nephrotoxic contrast media, hypotension from rapid ventricular pacing, and potential thromboembolization from catheter manipulation in a calcified aorta. Data comparing AKI following SAVR and TAVR is scarce. Similarly to SAVR, AKI has consistently been reported following TAVR with incidences ranging from $8.3 \%$ to $42 \%$ depending on the patient population; ${ }^{40,41}$ moreover AKI has been identified as a major determinant of CKD and mortality. ${ }^{42}$ AKI after SAVR and TAVR have been compared in the context of preoperative CKD by Bagur et al. who, in a study of 317 patients, showed that in patients with baseline CKD, AKI was more likely to occur following SAVR (26\% vs. 9\%). No study has so far directly compared AKI following TAVR and SAVR in patients with normal baseline renal function. The risk of developing AKI should be taken into consideration and weighed along with the other factors contributing to the decision making of the optimal therapeutic option of TAVR versus SAVR; however based on our data, the risk of AKI after SAVR is much lower than previously thought. This information supports the results of the PARTNER 1 and STACCATO prospective randomized clinical trials comparing TAVR and SAVR in high risk patients with AS, both of which found no difference in the incidence of postoperative AKI between the two groups. ${ }^{43-46}$

In addition to these trials, a number of retrospective observation studies reported, among other outcomes of interest, similar incidences of AKI in SAVR and TAVR patients. ${ }^{47,48}$

In conclusion, in our cohort, AKI was not found to be an independent predictor of short- or long-term mortality and its incidence was much lower than what has been reported after both on-CPB cardiac surgeries and TAVR. These results suggest that, from a purely renal perspective, SAVR does not confer an increased risk of AKI compared to TAVR for those patients with baseline borderline kidney function. Careful recognition of patients at risk of AKI is warranted for a better preoperative renal optimization.

\section{LIMITATIONS}

Our study is limited in that it is a single-center study and is retrospective in nature. In addition, because SCr levels were systematically ordered for all patients only during the first 48 hours whereas afterwards it was at the discretion of the care provider, we are unable to exclude the possibility that we might have missed a certain number of AKI cases due to unavailability of SCr. Concerning the study of kidney function over time, eGFR is a dynamic variable that varies over time and is affected by comorbidities and medications - thus the use of one value at each follow-up time over 12 months is not an exact measure of kidney 
function, but rather an estimation. However, we believe that serial results provide a very accurate description of an individual's renal function, and this limitation is minor.

\section{Supplementary Material}

Refer to Web version on PubMed Central for supplementary material.

\section{References}

1. Nishimura RA, Otto CM, Bonow RO, et al. 2014 AHA/ACC guideline for the management of patients with valvular heart disease: A report of the American College of Cardiology/American Heart Association task force on practice guidelines. J Am Coll Cardiol. 2014; 63(22):e57-e185. [PubMed: 24603191]

2. Vahanian A, Aflieri O, Andreotti F, et al. Guidelines on the management of valvular heart disease (version 2012): The joint task force on the management of valvular heart disease of the European Society of Cardiology (ESC) and the European Association of Cardio-Thoracic Surgery (EACTS). Eur Heart J. 2012; 33:2451-2496. [PubMed: 22922415]

3. Hobson CE, Yavas S, Segal MS, et al. Acute kidney injury is associated with increased long-term mortality after cardiothoracic surgery. Circulation. 2009; 119(18):2444-2453. [PubMed: 19398670]

4. Parolari A, Pesce LL, Pacini D, et al. Risk factors for perioperative acute kidney injury after adult cardiac surgery: Role of perioperative management. Ann Thorac Surg. 2012; 93(2):584-591. [PubMed: 22269725]

5. Bellomo R, Ronco C, Kellum JA, et al. Acute renal failure- Definition, outcome measures, animal models, fluid therapy and information technology needs the Second International Consensus Conference of the Acute Dialysis Quality Initiative (ADQI) Group. Crit Care. 2004; 8(4):R204 R212. [PubMed: 15312219]

6. Mehta RL, Kellum JA, Shah SV, et al. Acute kidney injury network: Report of an initiative to improve outcomes in acute kidney injury. Crit Care. 2007; 11:R31. [PubMed: 17331245]

7. Englberger L, Suri RM, Li Z, et al. Clinical accuracy of RIFLE and acute kidney injury network (AKIN) criteria for acute kidney injury in patients undergoing cardiac surgery. Crit Care. 2011; 15(1):R16. [PubMed: 21232094]

8. Thakar CV, Worley S, Arrigain S, et al. Influence of renal dysfunction on mortality after cardiac surgery: Modifying effect of preoperative renal function. Kidney Int. 2005; 67(3):1112-1119. [PubMed: 15698452]

9. Grayson AD, Khater M, Jackson M, et al. Valvular heart operation is an independent risk factor for acute renal failure. Ann Thorac Surg. 2003; 75(6):1829-1835. [PubMed: 12822624]

10. Brown JR, Kramer RS, Coca SG, et al. Duration of acute kidney injury impacts long-term survival after cardiac surgery. Ann Thorac Surg. 2010; 90(4):1142-1148. [PubMed: 20868804]

11. Elhmidi Y, Bleiziffer S, Deutsch MA, et al. Acute kidney injury after transcatheter aortic valve implantation: Inci-dence, predictors and impact on mortality. Arch Cardio-vasc Dis. 2014; 107(2): 133-139.

12. O’Connor GT, Plume SK, Olmstead EM, et al. A regional prospective study of in-hospital mortality associated with coronary artery bypass grafting. The Northern New England Cardiovascular Disease Study Group. JAMA. 1991; 266(6):803-809. [PubMed: 1907669]

13. Thakar CV, Liangos O, Yared JP, et al. ARF after open-heart surgery: Influence of gender and race. Am J Kidney Dis. 2003; 41(4):742-751. [PubMed: 12666060]

14. Dasta JF, Kane-Gill SL, Durtschi AL, et al. Costs and outcomes of acute kidney injury (AKI) following cardiac surgery. Nephrol Dial Transplant. 2008; 23(6):1970-1974. [PubMed: 18178605]

15. Lassnigg A, Schmidlin D, Mouhieddine M, et al. Minimal changes of serum creatinine predict prognosis in patients after cardiothoracic surgery: A prospective cohort study. J Am Soc Nephrol. 2004; 15(6):1597-1605. [PubMed: 15153571]

16. Lok CE, Austin PC, Wang H, et al. Impact of renal insufficiency on short- and long-term outcomes after cardiac surgery. Am Heart J. 2004; 148(3):430-438. [PubMed: 15389229] 
17. National Kidney F. K/DOQI clinical practice guidelines for chronic kidney disease: Evaluation, classification, and stratification. Am J Kidney Dis. 2002; 39:S1-266. [PubMed: 11904577]

18. Robert AM, Kramer RS, Dacey LJ, et al. Cardiac surgery-associated acute kidney injury: A comparison of two con-sensus criteria. Ann Thorac Surg. 2010; 90(6):1939-1943. [PubMed: 21095340]

19. Karkouti K, Wijeysundera DN, Yau TM, et al. Acute kidney injury after cardiac surgery: Focus on modifiable risk factors. Circulation. 2009; 119(4):495-502. [PubMed: 19153273]

20. Bove T, Calabro MG, Landoni G, et al. The incidence and risk of acute renal failure after cardiac surgery. J Cardiothorac Vasc Anesth. 2004; 18(4):442-445. [PubMed: 15365924]

21. Murashita T, Greason KL, Suri RM, et al. Aortic valve replacement for severe aortic valve stenosis in the non-agenarian patient. Ann Thorac Surg. 2014; 98(5):1593-1597. [PubMed: 25200726]

22. D’Onofrio A, Cruz D, Bolgan I, et al. RIFLE criteria for cardiac surgery-associated acute kidney injury: Risk factors and outcomes. Congest Heart Fail. 2010; 16(1):S32-S36. [PubMed: 20653709]

23. Kappetein AP, Head SJ, Genereux P, et al. Updated standardized endpoint definitions for transcatheter aortic valve implantation: The valve academic research consor-tium- 2 consensus document. J Thorac Cardiovasc Surg. 2013; 145(1):6-23. [PubMed: 23084102]

24. Lazar HL. The use of angiotensin-converting enzyme inhibitors in patients undergoing coronary artery bypass graft surgery. Vascul Pharmacol. 2005; 42:119-123. [PubMed: 15792929]

25. Devbhandari MP, Balasubramanian SK, Codispoti M, et al. Preoperative angiotensin-converting enzyme inhibition can cause severe post CPB vasodilation-Current UK opinion. Asian Cardiovasc Thorac Ann. 2004; 12:346-349. [PubMed: 15585706]

26. Kwapisz MM, Muller M, Schindler E, et al. The effect of intravenous quinaprilat on plasma cytokines and hemodynamic variables during cardiac surgery. J Cardiothorac Vasc Anesth. 2004; 18:53-58. [PubMed: 14973800]

27. Patel NN, Rogers CA, Angelini GD, et al. Pharmacological therapies for the prevention of acute kidney injury following cardiac surgery: A systematic review. Heart Fail Rev. 2011; 16:553-567. [PubMed: 21400231]

28. Landoni G, Biondi-Zoccai GG, Marino G, et al. Fenoldopam reduces the need for renal replacement therapy and in-hospital death in cardiovascular surgery: A meta-analysis. $\mathbf{J}$ Cardiothorac Vasc Anesth. 2008; 22:27-33. [PubMed: 18249327]

29. Brater DC. Diuretic therapy. N Engl J Med. 1998; 339(6):387-395. [PubMed: 9691107]

30. Mariscalco G, Cottini M, Dominici C, et al. The effect of timing of cardiac catheterization on acute kidney injury after cardiac surgery is influenced by the type of operation. Int J Cardiol. 2014; 173:46-54. [PubMed: 24602318]

31. Greason KL, Englberger L, Suri RM, et al. Safety of same-day coronary angiography in patients undergoing elective aortic valve replacement. Ann Thorac Surg. 2011; 91:1791-1797. [PubMed: 21619975]

32. Andersen ND, Williams JB, Fosbol EL, et al. Cardiac catheterization within 1 to 3 days of proximal aortic surgery is not associated with increased postoperative acute kidney injury. $\mathbf{J}$ Thorac Cardiovasc Surg. 2012; 143:1404-1410. [PubMed: 22341657]

33. Kieran NE, Rabb H. Immune responses in kidney preservation and reperfusion injury. J Investig Med. 2004; 52(5):310-314.

34. Liu KD, Brakeman PR. Renal repair and recovery. Crit Care Med. 2008; 36(suppl 4):S187-S192. [PubMed: 18382192]

35. Abbate M, Remuzzi G. Acceleration of recovery in acute renal failure: From cellular mechanisms of tubular repair to innovative targeted therapies. Ren Fail. 1996; 18(3):377-388. [PubMed: 8827982]

36. Bent P, Tan HK, Bellomo R, et al. Early and intensive continuous hemofiltration for severe renal failure after cardiac surgery. Ann Thorac Surg. 2001; 71(3):832-837. [PubMed: 11269461]

37. Schiffl H, Lang SM, Fischer R, et al. Daily hemodialysis and the outcome of acute renal failure. N Engl J Med. 2002; 346(5):305-310. [PubMed: 11821506] 
38. Elahi MM, Lim MY, Joseph RN, et al. Early hemofiltration improves survival in post-cardiotomy patients with acute renal failure. Eur J Cardiothorac Surg. 2004; 26:1027-1031. [PubMed: 15519198]

39. Luckraz H, Gravenor MB, George R, et al. Long and short-term outcomes in patients requiring continuous renal replacement therapy post cardiopulmonary bypass. Eur J Cardiothorac Surg. 2005; 27:906-909. [PubMed: 15848334]

40. Genereux P, Kodali SK, Green P, et al. Incidence and effect of acute kidney injury after transcatheter aortic valve replacement using the new valve academic research consortium criteria. Am J Cardiol. 2013; 111(1):100-105. [PubMed: 23040657]

41. Saia F, Ciuca C, Taglieri N, et al. Acute kidney injury following transcatheter aortic valve implantation: Incidence, predictors and clinical outcome. Int J Cardiol. 2013; 168:1034-1040. [PubMed: 23164594]

42. Nguyen TC, Babaliaros VC, Razavi SA, et al. Impact of varying degrees of renal dysfunction on transcatheter and surgical aortic valve replacement. J Thorac Cardiovasc Surg. 2013; 146(6):1399_ 1407. [PubMed: 24075566]

43. Leon MB, Smith CR, Mack MJ, et al. Transcatheter aortic-valve implantation in patients who cannot undergo surgery. N Engl J Med. 2010; 363:1597-16074. [PubMed: 20961243]

44. Smith CR, Leon MB, Mack MJ, et al. Transcatheter versus surgical aortic-valve replacement in high-risk patients. N Engl J Med. 2011; 364:2187-2198. [PubMed: 21639811]

45. Makkar RR, Fontana GP, Jilaihawi H, et al. Transcatheter aortic-valve replacement for inoperable severe aortic stenosis. N Engl J Med. 2012; 366:1696-1704. [PubMed: 22443478]

46. Nielsen HH, Klaaborg KE, Nissen H, et al. A prospective, randomised trial of transapical transcatheter aortic valve implantation vs. surgical aortic valve replacement in operable elderly patients with aortic stenosis: The STACCATO trial. EuroIntervention. 2012; 8:383-389. [PubMed: 22581299]

47. Tamburino C, Barbanti M, Capodanno D, et al. Comparison of complications and outcomes to one year of transcatheter aortic valve implantation versus surgical aortic valve replacement in patients with severe aortic stenosis. Am J Cardiol. 2012; 109:1487-1493. [PubMed: 22356793]

48. Cao C, Ang SC, Indraratna P, et al. Systematic review and meta-analysis of transcatheter aortic valve implantation versus surgical aortic valve replacement for severe aortic stenosis. Ann Cardiothorac Surg. 2013; 2(1):10-23. [PubMed: 23977554] 


\section{0}
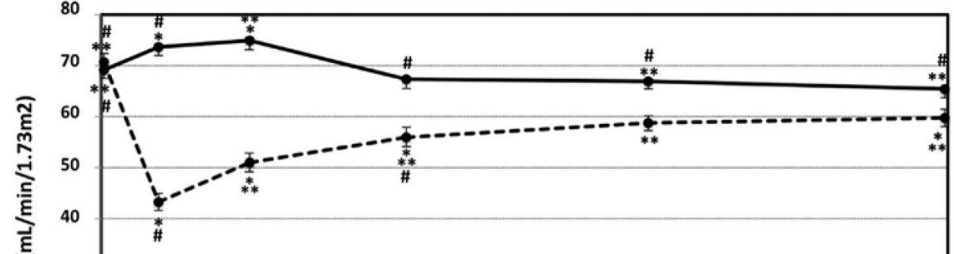

造 30

20

10

0

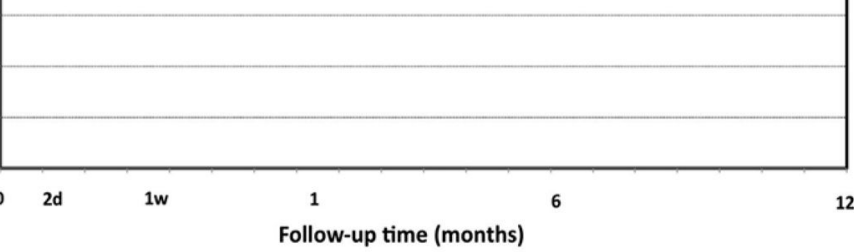

Number at follow-up

$\rightarrow$ No AKI $19451887 \quad 1583$

374

152

53

19

26

\section{Figure 1.}

Dynamics of kidney function following aortic valve replacement for patients with AKI (dotted line) and without AKI (solid line). ( indicates $\mathrm{p}<0.05$ vs. time 0 ; indicates $\mathrm{p}<0.05$ vs. 2 days; \# indicates $\mathrm{p}<0.05$ vs. 1 week). AKI, acute kidney injury. 


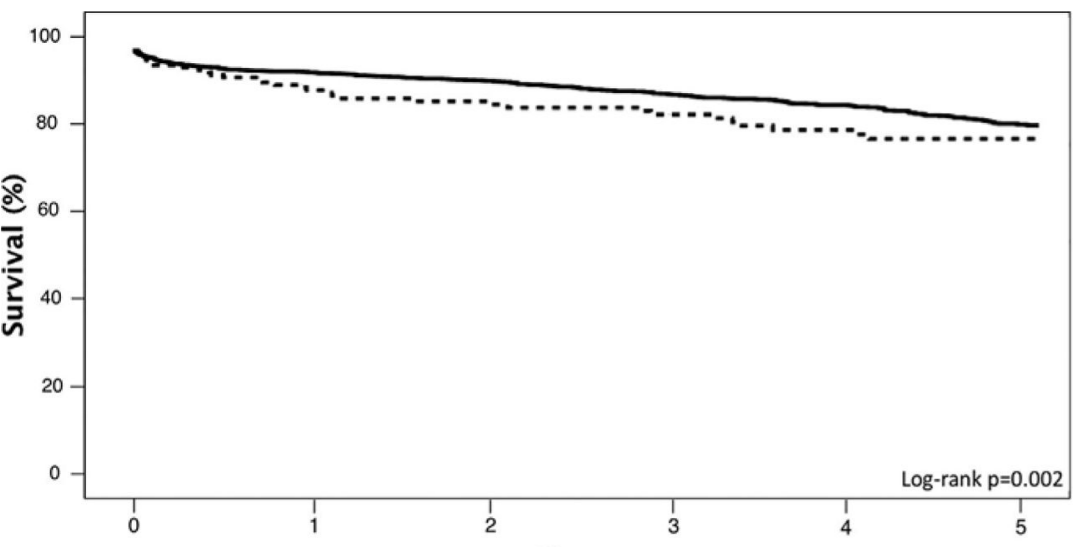

Number at Risk

Years

$\boldsymbol{r}$ No AKI

$=$ AKI
1775

147
1445

124
1182

105
997

80
811

65

Figure 2.

Kaplan-Meier survival estimates for patients with AKI (dotted line) and without AKI (solid line). AKI, acute kidney injury. 
TABLE 1

\section{AKI AKIN Definition}

\begin{tabular}{|c|c|c|}
\hline & Serum Creatinine (SCr) Criteria & Urine Output (U/O) Criteria \\
\hline Stage 1 & $\begin{array}{l}\text { Increase in SCr to } 150-199 \%(1.5-1.99 \times \text { increased compared with } \\
\text { baseline) OR Increase of } 0.3 \mathrm{mg} / \mathrm{dL}(26.4 \mathrm{mmol} / \mathrm{L})\end{array}$ & $\mathrm{U} / \mathrm{O}<0.5 \mathrm{~mL} / \mathrm{kg} / \mathrm{h}$ for $>6$ but $<12-$ hours \\
\hline Stage 2 & Increase in SCr to 200-299\% (2.0-2.99 increase compared with baseline) & $\mathrm{U} / \mathrm{O}<0.5 \mathrm{~mL} / \mathrm{kg} / \mathrm{h}$ for $>12$ but $<24$ hours \\
\hline Stage 3 & $\begin{array}{l}\text { Increase in } \mathrm{SCr} \text { to } 300 \%(3 \text { increase compared with baseline) or SCr of } 4.0 \\
\mathrm{mg} / \mathrm{dL}(354 \mathrm{mmol} / \mathrm{L}) \text { with an acute increase of at least } 0.5 \mathrm{mg} / \mathrm{dL}(44 \\
\mathrm{mmol} / \mathrm{L})\end{array}$ & $\mathrm{U} / \mathrm{O}<0.3 \mathrm{~mL} / \mathrm{kg} / \mathrm{h}$ for $>24 \mathrm{~h}$ OR Anuria for 12 hours \\
\hline
\end{tabular}

AKIN, acute kidney injury network; SCr, serum creatinine; U/O, urine output. 
TABLE 2

\section{Baseline Characteristics}

\begin{tabular}{lccc}
\hline & AKIS $(\mathbf{n}=\mathbf{1 9 4 5})$ & AKI R $(\mathbf{n}=\mathbf{1 8 1})$ & p-Value \\
\hline Age (years, mean SD) & 70.514 .4 & 72.014 .1 & 0.167 \\
Female gender, n (\%) & $831(43)$ & $80(44)$ & 0.754 \\
BMI (kg/m², mean SD) & 285.7 & 296.4 & 0.008 \\
LVEF (\%, mean SD) & 5113 & 5113 & 0.725 \\
Diabetes, n (\%) & $385(20)$ & $47(26)$ & 0.048 \\
PVD, n (\%) & $122(6.3)$ & $17(9.4)$ & 0.115 \\
CVD, n (\%) & $188(9.7)$ & $25(14)$ & 0.091 \\
COPD, n (\%) & $186(9.6)$ & $27(15)$ & 0.022 \\
Cr (mg/dL, mean SD) & 1.10 .58 & 1.00 .36 & 0.113 \\
eGFR (mL/min/1.732, mean SD) & 69.251 .5 & 70.723 .0 & 0.374 \\
Cardiac catheterization interval 5 days, n (\%) & $589(30)$ & $59(33)$ & 0.555 \\
\hline
\end{tabular}

AKI, acute kidney injury; BMI, body mass index; LVEF, left ventricle ejection fraction; PVD, peripheral vascular disease; CVD, cerebrovascular disease; COPD, chronic obstructive pulmonary disease; $\mathrm{Cr}$, creatinine; eGFR, estimated glomerular filtration rate. 


\section{TABLE 3}

Operative Characteristics

\begin{tabular}{lccc}
\hline & AKIS (n = 1945) & AKIR $(\mathbf{n}=\mathbf{1 8 1})$ & p-Value \\
\hline Operative priority, n (\%) & & & 0.531 \\
$\quad$ Elective & $1681(87)$ & $151(83)$ & \\
Urgent & $239(12)$ & $27(15)$ & \\
$\quad$ Emergent & $24(1.2)$ & $3(1.7)$ & \\
Mechanical valve, n (\%) & $187(9.6)$ & $13(7.2)$ & 0.273 \\
Previous cardiac & $374(19)$ & $35(19)$ & 1.000 \\
surgery, n (\%) & 232.5 & 232.6 & \\
Valve size (mean SD) & & & 0.402 \\
XC time (min, mean SD) & 6220 & 6421 & 0.396 \\
CPB time (min, mean SD) & 8933 & 9035 & 0.720 \\
Lowest temp ( ${ }^{\circ} \mathrm{C}$, mean SD) & 32.59 .6 & 32.22 .2 & 0.339 \\
PRBC transfusion, n (\%) & $595(31)$ & $73(40)$ & 0.007 \\
PRBC (units, mean SD) & 0.781 .5 & 1.11 .4 & 0.015 \\
Cell saver (n, mean SD) & 2.51 .2 & 2.51 .0 & 0.901 \\
\hline
\end{tabular}

AKI, acute kidney injury; XC time, cross-clamp time; CPB time, cardiopulmonary bypass time; PRBC, packed red blood cells. 
TABLE 4

Post-Operative Outcomes

\begin{tabular}{lccc}
\hline & AKIS (n = 1945) & AKIR (n = 181) & p-Value \\
\hline AKIN classification & & & N/A \\
$\quad$ Stage 1 AKI, n (\%) & N/A & $121(67)$ & \\
$\quad$ Stage 2 AKI, n (\%) & N/A & $42(23)$ & \\
$\quad$ Stage 3 AKI, n (\%) & N/A & $7(3.9)$ & N/A \\
Need for dialysis, n (\%) & $29(1.5)$ & $3(1.7)$ & 0.750 \\
Debilitating stroke, n (\%) & $13(0.7)$ & $3(1.7)$ & 0.150 \\
Sternal wound infection, n (\%) & $10(0.5)$ & $4(2.2)$ & 0.026 \\
Sepsis, n (\%) & $66(3.4)$ & $5(2.8)$ & 0.682 \\
Reoperation for bleeding, n (\%) & $577(30)$ & $60(33)$ & 0.351 \\
PRBC transfusion, n (\%) & 0.92 .2 & 1.02 .2 & 0.477 \\
PRBC (units, mean SD) & 2.63 .7 & 4.25 .3 & $<0.001$ \\
ICULOS, days (mean SD) & 109.3 & 1513 & $<0.001$ \\
THLOS, days (mean SD) & $560(29)$ & $51(28)$ & 0.864 \\
Mechanical ventilation >24 hours, n (\%) & $65(3)$ & $19(11)$ & $<0.001$ \\
Readmission to ICU, n (\%) & $30(1.5)$ & $5(2.8)$ & 0.215 \\
In-hospital mortality, n (\%) & $338(17)$ & $45(25)$ & 0.012 \\
Cumulative mortality, n (\%) & & & \\
\hline
\end{tabular}

AKI, acute kidney injury; PRBC, packed red blood cells; ICULOS, intensive care unit length of stay; THLOS, total hospital length of stay; ICU, intensive care unit.

p-value based on Mann-Whitney U test. 
TABLE 5

Multivariable Predictors of Acute Kidney Injury After Surgical Aortic Valve Replacement

\begin{tabular}{lcccc}
\hline Variable & Odds Ratio & Lower 95\% CI & Higher 95\% CI & p-Value \\
\hline BMI & 1.032 & 1.007 & 1.057 & 0.013 \\
COPD & 1.530 & 0.985 & 2.376 & 0.058 \\
CVD & 1.454 & 0.926 & 2.284 & 0.104 \\
Diabetes & 1.235 & 0.879 & 1.775 & 0.254 \\
Intraoperative PRBC transfusion & 1.094 & 1.006 & 1.191 & 0.037 \\
\hline
\end{tabular}

AKI, acute kidney injury; BMI, body mass index; COPD, chronic obstructive pulmonary disease; CVD, cerebrovascular disease; PRBC, packed red blood cells; CI, confidence interval. 
TABLE 6

Predictors of All Cause Mortality from Multivariate Cox Regression Survival Analysis

\begin{tabular}{lccrr}
\hline & Adjusted Hazard Ratio & Lower 95\% CI & Higher 95\% CI & p-Value \\
\hline Age & 1.050 & 1.037 & 1.062 & $<0.001$ \\
CVD & 1.465 & 1.079 & 1.988 & 0.014 \\
COPD & 1.801 & 1.349 & 2.406 & $<0.001$ \\
Preoperative LVEF & 0.985 & 0.978 & 0.993 & $<0.001$ \\
Intraoperative PRBC transfusion & 1.319 & 1.030 & 1.689 & 0.028 \\
Postoperative need for dialysis & 5.091 & 1.554 & 16.673 & 0.007 \\
ICULOS & 1.024 & 1.006 & 1.042 & 0.007 \\
Postoperative readmission to ICU & 2.715 & 1.852 & 3.979 & $<0.001$ \\
\hline
\end{tabular}

CVD, cerebrovascular disease; COPD, chronic obstructive pulmonary disease; LVEF, left ventricular ejection fraction; PRBC, packed red blood cells; ICULOS, intensive care unit length of stay; ICU, intensive care unit; CI, confidence interval. 